\title{
Pausas preenchidas na fala espontânea de pacientes com esquizofrenia: comparação entre pacientes e não pacientes
}

José Carlos Costa (UFMG)*

https://orcid.org/0000-0001-8265-4195

\section{Resumo:}

O objetivo deste trabalho é fazer uma comparação entre a frequência de ocorrência e duração de pausas preenchidas na fala espontânea de pessoas com esquizofrenia. A hipótese é de que haveria mais pausas e de maior duração na fala de pessoas com esquizofrenia do que no de pessoas sem essa condição. Metodologicamente, utilizamos 6 áudios e suas respectivas transcrições do C-ORAL-ESQ (FERRARI, ROCHA, em construção) e 6 do C-ORAL -BRASIL (RASO, MELLO, 2012), a partir dos quais foram demarcadas as pausas preenchidas. Para extração, tratamento dos dados e análise estatística foram utilizadas bibliotecas da linguagem Python. Os resultados mostram que pacientes com esquizofrenia realizaram maior quantidade de pausas e com maior duração, quando comparados por corpora, e pausas geralmente maiores quando comparados individualmente, com resultados estatisticamente relevantes no teste $U$ de Mann Whitney $(\mathrm{p}<0,05)$ para a diferença registrada na duração das pausas. Esses resultados sugerem que pacientes com esquizofrenia hesitam mais na fala espontânea do que pessoas sem esquizofrenia, mas ainda é necessário estudar o contexto no qual cada uma das 94 pausas encontradas ocorreram para categorizá-las como de fato uma disfluência.

Palavras-chave: Esquizofrenia; Pausas preenchidas; Python.

\section{Abstract:}

\section{Filled pauses in schizophrenic spontaneous speech: comparison between patients and no patients}

The aim of this research is to make a comparison between frequency and length of filled pauses in schizophrenic spontaneous speech. We hypothesize that people with schizophrenia would make more filled pauses and with

Doutorando em Estudos Linguísticos pela Universidade Federal de Minas Gerais. Lattes: http://lattes. cnpq.br/3336031546876937. email: carlosjuniorcosta1@gmail.com 
longer duration than non-schizophrenic people. Our methods consist in extracting filled pauses out of 6 audios transcriptions from C-ORAL-ESQ (FERRARI, ROCHA, in construction) and C-ORAL-BRASIL, data treatment and statistical analysis in Python language environment. The results show that schizophrenic patients made more filled pauses and with longer duration than non-schizophrenics - in the comparison across the whole subcorpora - and usually more pauses with longer duration when patients were compared individually. There are significant statistical results in the U Mann Whitney test $(p<0,05)$ regarding length of pauses. We claim that patients with schizophrenia appear to hesitate more than non-schizophrenics in spontaneous speech, although it may be necessary to take into account the context of each of 94 filled pauses found before claiming those as in fact disfluencies.

Keywords: Schizophrenia; Filled pauses; Python.

\section{Introdução}

O objetivo deste trabalho é apresentar uma comparação entre pausas preenchidas na fala espontânea de pessoas com esquizofrenia e sem esquizofrenia ${ }^{1}$. A hipótese deste artigo é que pacientes com esquizofrenia realizariam mais pausas preenchidas e de maior duração do que pacientes sem esquizofrenia na fala espontânea. Essa suposição parte de trabalhos que já analisaram pausas na fala desses pacientes (ALPERT et al, 1997; FELDSTEN, 1963; MATSUMOTO et al, 2013; entre outros) e já constataram maior quantidade de pausas - preenchidas ou silenciosas - na fala desses pacientes do que de pessoas sem esquizofrenia.

Na seção 1, são apresentados alguns problemas linguísticos recorrentes relativos à fala de pessoas com esquizofrenia, com ênfase no tema deste artigo, que são as pausas preenchidas. Na seção 2, são detalhados os corpora utilizados e os procedimentos metodológicos utilizados para extração dos dados utilizados. Na seção 3, são apresentados os resultados obtidos na comparação entre

1 Trata-se de uma comparação preliminar, uma vez que um dos corpora utilizados, C-ORALESQ (FERRARI, ROCHA, em construção) está em processo de construção e revisão. pausas preenchidas e, na seção 4, por fim, as considerações finais deste trabalho.

\section{Pressupostos teóricos}

A esquizofrenia é uma doença mental caracterizada pela presença de uma ou mais de uma das ocorrências a seguir: delírios, alucinações, pensamento e fala com distúrbios, comportamento motor muito desorganizado ou anormal - sintomas positivos - e sintomas negativos (DSM-5, 2013, p.87).

É importante destacar que sintomas positivos refletem o excesso ou distorção do funcionamento normal, enquanto os negativos dizem respeito à ausência de características que normalmente possuem os indivíduos sem essa doença (DSM-V, 2013, p. 87). Os sintomas negativos mais comuns são a alogia, caracterizada pela pouca quantidade de fala ou de seu conteúdo; anedonia, que reflete a dificuldade do paciente sentir e expressar prazer, intimidade ou ter contatos sociais; embotamento afetivo; apatia e déficit de atenção (ANDREASEN, 1990, p. 616).

Existem vários estudos que contemplam variados problemas relativos à linguagem utilizada por pacientes com esquizofrenia. $\mathrm{Na}$ Fonética e Fonologia, já foi relato, por 
exemplo, monotonia prosódica (COVIGTON, 2005); rima e aliteração na fala de pacientes (ANDREASEN, 1986); prosódia afetiva - que relata dificuldades dos pacientes em processar e expressar alguns tipos de sentimentos (LIN et al, 2018), entre outros.

Na Morfologia e Sintaxe, já foi constatado, por exemplo, simplificação sintática de pacientes (MORICE, INGRAN, 1983), sintaxe de recepção transtornada (CONDRAY et al, 2002).

$\mathrm{Na}$ área da Semântica e do Léxico, já foram verificados fenômenos como a glossomania, um tipo de compulsão em utilizar palavras de um mesmo campo semântico (ANDREASEN, 1986; COVINGTON et al, 2005); bloqueio lexical (parada súbita na fala) (ANDREASEN, 1986; COVINGTON et al, 2005) e salada de palavras (COVINGTON et al, 2005).

Já a área da Pragmática, que de alguma forma também se relaciona à competência discursiva dos pacientes com esquizofrenia, possui quantidade bastante significativa de trabalhos (STEUBER, 2011). Entre os diversos fenômenos registrados, destacamos excesso ou pobreza de fala/conteúdo (ANDREASEN, 1986; LIDDLE et al, 2002); excesso ou pobreza de referência: excesso de referência não verbal, excesso de referência de si mesmo, excesso ou falta de informação pressuposta do interlocutor (ANDREASEN, 1986; COVINGTON et al, 2006); falta de lógica (ANDREASEN, 1986; LIDDLE et al, 2006), repetição não esperada (LIDDLE et al, 2002), perseverança em tópicos (ANDREASEN, 1986) e ecolalia (ANDREASEN, 1986).

0 estudo das pausas - preenchidas ou não - também ocupa lugar de destaque na literatura sobre problemas relativos à linguagem de pacientes com esquizofrenia. Nas subseções 1.1 e 1.2 a seguir, problematizamos e destacamos alguns estudos das pausas na fala de pacientes com esquizofrenia.

\subsection{Pausas}

A pausa é uma característica comum na fala espontânea, tanto na forma de intervalos silenciosos quanto vocalizados. No intervalo silencioso, o falante não produz nenhuma vocalização durante algum período. Nos vocalizados, o falante prolonga, por alguma razão, algum tipo de segmento geralmente sem significado lexical (ESPOSITO et al, 2006, p.542).

Em termos acústicos, a duração da pausa é bastante controversa. Esposito et al (2007, p. 545), em seu estudo que investiga as pausas silenciosas de crianças e adultos em narrativas espontâneas, considera o limite mínimo de 120 ms ou mais para pausas silenciosas. Çokal et al, (2019, p. 4) que estudam o padrão das pausas em pacientes com esquizofrenia com ou sem desordem do pensamento, estabelecem que pausas menores do que $250 \mathrm{~ms}$, independentemente do tipo, são "espaços fonatórios ligados ao ciclo de respiração" que são muito difíceis de serem percebidos. Já Boer et al (2020, p. 8), os quais investigam diversos problemas linguísticos e sua relação com os sintomas positivos ou negativos da esquizofrenia, consideram pausas silêncios com mais de 200 ms. Dessa forma, é notório que é necessário considerar o tipo de pausa a ser pesquisado e estabelecer um limite mínimo de tempo para ser considerado uma pausa, uma vez que não há esse limite pré-definido de forma categórica na literatura.

Tampouco há consenso a respeito das causas das pausas, independente se silenciosas ou preenchidas. O'Connell e Kowal (1983, p.222), por exemplo, defendem que fatores como respiração, interrupção, complexidade sintática, disponibilidade de itens lexicais na mente do falante, ênfase e sentimentos como raiva, confusão, ansiedade e vergonha podem estar relacionados com 
as pausas. Dessa forma, uma pausa é comumente associada a um conjunto de fatores, e é preciso levar em conta essa complexidade para estudá-la mais adequadamente.

É possível que as pausas também estejam relacionadas ao controle do fluxo de informação. Butterworth (1980), por exemplo, defende que um falante utiliza a pausa para permitir que o ouvinte processe a informação anterior no fluxo da fala. Já no caso específico das pausas preenchidas, há a associação desse fenômeno ao ganho de tempo pelo falante (BORTFELD et al, 2001) e também à manutenção do turno de fala, já que uma pausa silenciosa poderia favorecer à tomada de turno por outro falante (OGATA et al, 2009).

\subsection{Pausas preenchidas e fala de pessoas com esquizofrenia}

Apesar de claramente separadas em classes maiores, pausas silenciosas e preenchidas, não é raro que alguns autores as agrupem ao fazer generalizações a respeito da fala de pacientes com esquizofrenia. Alpert et al (1997, p.171), por exemplo, destacam que pacientes com sintomas negativos mais acentuados usualmente realizam pausas preenchidas ou não - com maior duração, tanto em relação a pacientes com menos sintomas negativos quanto a pessoas sem esquizofrenia.

Em um antigo estudo de Feldstein e Jaffe (1963), grupos compostos por 30 pacientes com esquizofrenia e 30 sem esquizofrenia foram comparados em relação à frequência de ocorrência de pausas preenchidas. No experimento, os participantes deveriam observar quatro figuras, duas descritas como "afetivas" e duas como "não afetivas" ao longo de um minuto. Imediatamente depois, cada indivíduo deveria contar do que se lembrava da história remetida pela figu- ra. Os resultados indicaram que pacientes com esquizofrenia realizaram mais pausas preenchidas do que o grupo controle. Para os autores, todos os indivíduos possuem uma "distribuição" de respostas comportamentais e linguísticas que são acionadas em determinados ambientes (p.778). Nos pacientes com esquizofrenia, a maior frequência de pausas preenchidas indicaria uma distribuição linguística "que é mais ampla e possui diferente probabilidades de estruturas do que a distribuição linguística de pessoas sem esquizofrenia" (p.778). Entretanto, os autores não detalham a chamada distribuição linguística e tampouco fornecem medidas em relação à duração das pausas entre os dois grupos.

Ocorre que a maior frequência de pausas preenchidas em pacientes com esquizofrenia tampouco é um consenso. Matsumoto et al (2013), por exemplo, submeteram 6 pacientes com esquizofrenia e seis pessoas sem esquizofrenia a um teste que consistia em descrever 7 placas de Rorschach vistas por meio de um espelho. Ao longo de $21 \mathrm{mi}-$ nutos, sendo 3 para cada placa, os participantes falavam o que eles viam nas placas. Foi registrado que pacientes sem esquizofrenia realizaram quantidade muito menor de pausas no experimento do que pessoas sem esquizofrenia. Segundo os autores, realizar pausas preenchidas significaria monitorar mais o discurso em relação a erros de produção e outras anomalias, e o fato de os pacientes com esquizofrenia terem realizado menos pausas do que pessoas sem essa doença indicaria menor monitoramento discursivo desses pacientes.

\section{Metodologia}

Esta seção detalha a metodologia empregada neste trabalho. Nas subseções a seguir, detalhamos os corpora utilizados e os pro- 
cedimentos empregados na aferição e comparação das pausas preenchidas.

\subsection{Corpora e dados utilizados}

Os corpora utilizados neste trabalho, como já mencionado, foram o C-ORAL-ESQ (FERRARI, ROCHA, RASO, em construção) representativo da fala de pacientes com esquizofrenia, e C-ORAL-BRASIL (RASO, MELLO, 2012), representativo do português brasileiro falado. Foram escolhidos seis áudios e suas respectivas transcrições do C-ORAL-ESQ - as únicas que até então estavam disponíveis - e 6 áudios do minicorpus de monólogos do C-ORAL-BRASIL.

No C-ORAL-ESQ o contexto de interação é o de consulta psiquiátrica entre médico e paciente, além de ocasionais terceiros, que não estavam presentes nos áudios selecionados. No C-ORAL-BRASIL, por sua vez, o contexto de interação é o de uma pessoa que conta uma história de sua vida após ser instigado por outro participante, que apenas raramente faz intervenções. Apesar de um corpus não ser o corpus controle de outro, e possuírem contextos de interação diferentes, ambos são representativos de suas populações. Por representativo entende-se um corpus que contemple a distribuição estratificada de sua população e de fenômenos linguísticos para os quais esse corpus visa a ser representativo. Isso significa que as amostras presentes no corpus devem representar a variedade linguística e social de sua população (BIBER, 1993, 242-246).

A partir desses corpora originais, construímos o que denominamos subcorpora, um para cada corpus, para que as amostras que retiramos não se confundam com a integridade dos corpora originais.

O subcorpus do C-ORAL-ESQ utilizado era composto por 4 homens e 2 mulheres, com idade entre 19 e 55 anos, além de esco- laridade média de 9 anos de estudo. 0 subcorpus do C-ORAL-BRASIL, por sua vez, era composto por 3 homens e 3 mulheres, idade entre 26 e dois participantes com mais de 60 anos, além de três pessoas com até o primário completo e três pessoas com até o terceiro grau completo ${ }^{2}$.

\subsection{Procedimentos}

Nos subcorpora utilizados, os áudios possuem alinhamento entre texto e fala, de forma que, quando se aciona o texto, também se escuta a fala do trecho. Esse alinhamento produz um arquivo de xml do qual é possível extrair a duração dos trechos alinhados.

Nesses áudios, as pausas preenchidas são marcadas pela etiqueta TMT (tomada de tempo). Nos xml disponibilizados ${ }^{3}$, essas pausas preenchidas não estão demarcadas, isto é, não é fornecido o tempo de sua duração, de forma que foi necessário localizar todas as etiquetas de TMT e demarcá-las antes de fazer qualquer comparação. Essas pausas não foram demarcadas anteriormente porque o critério para alinhamento nesses corpora é o enunciado, no qual as pausas estão, mas não apenas as pausas preenchidas em si. Dessa forma, apesar de ser possível averiguar que há pausas preenchidas em um enunciado, não é possível saber sua duração.

A localização das pausas foi realizada por meio da utilização da biblioteca Element Tree ${ }^{4}$, de tratamento de xml, além de um conjunto de bibliotecas de análise de dados em ambiente Python ${ }^{5}$. Essas bibliotecas

2 Os dados relativos à escolaridade são dados de forma diferente até esse momento de construção do corpus C-ORAL-ESQ.

3 http://www.c-oral-brasil.org/minicorpus_portugues_brasileiro_2018.html

4 https://docs.python.org/3/library/xml.etree. elementtree.html

5 https://colab.research.google.com/drive/1WnVEsHamy9eD8Zmwvrl7JKwsk095yT4j?us$\mathrm{p}=$ sharing 
permitem extrair dados dos xml dos áudios e montar uma tabela com todas as ocorrências de pausas preenchidas e os respectivos enunciados nos quais se encontram. A tabela a seguir exemplifica essa filtragem. Neste trabalho, apenas as pausas preenchidas interessam, de forma que o leitor deve ignorar outras etiquetas, pois não são importantes para esta análise.

Tabela 1 - Mapeamento de pausas preenchidas nos corpora

\begin{tabular}{c|c|c|c|c}
\hline index & Áudio & Ordem & Enunciados & $\begin{array}{c}\text { Pausas preenchidas } \\
\text { - TMT }\end{array}$ \\
\hline 1 & bfammn01_2018 & 2 & $\begin{array}{c}\text { \&he /=TMT= \&he /=TMT=o } \\
\text { negócio é seguinte } / /=\mathrm{COM}=\end{array}$ & 2 \\
\hline 5 & bfammn01_2018 & 6 & $\begin{array}{c}\text { \&he /=TMT= esse rapaz /=TOP= } \\
\text { \&he /=TMT= abriu um [/1]=SCA= } \\
\text { um claro dentro de uma mata } \\
\text { /=COB= pa fazer uma [/1]=SCA= } \\
\text { uma plantação /=COB= fazer \&u } \\
\text { [/1]=SCA= tipo [/1]=EMP= tipo } \\
\text { de lavoura /=COM=né //=AUX= }\end{array}$ \\
\hline
\end{tabular}

Fonte: Elaborado pelo autor

A Tabela 1 fornece a quantidade de pausas preenchidas em cada enunciado dos corpora utilizados em relação à frequência de ocorrência. Para obter a duração dessas pausas, foi necessário utilizar o xml e o áudio fornecido pelos corpora, localizar os enunciados da Tabela 1 e separar as pausas preenchidas, que são marcadas pela etiqueta TMT. Isso gera uma fronteira esquerda e direita na onda acústica, que é refletida no arquivo xml na forma de um tempo inicial e outro tempo final, a partir do qual é possível extrair a duração de cada unidade alinhada.

Nesse processo, foi considerada toda a extensão do segmento na onda acústica, independentemente de sua duração. Conforme já discutido na subseção 1.1, a duração mínima para uma pausa é bastante controversa, inclusive para pesquisadores da fala de pessoas com esquizofrenia. Neste trabalho, foram encontradas evidências de ocorrência de pausas preenchidas a partir de 181 ms no C-ORAL-ESQ e até 70 ms no C-ORAL-BRASIL. Por isso, não foi estabelecido um limite mínimo para pausas preenchidas.
Após transformadas em xml pelo WinPitch, as pausas foram novamente tratadas em ambiente Python para análise, a qual é realizada na seção de resultados a seguir.

\section{Resultados}

Os resultados são separados em duas subseções. Em 3.1, é discutida a comparação de frequência e duração na comparação por subcorpus, isto é, de todos os dados do C-ORAL -ESQ com todos os dados do C-ORAL-BRASIL utilizados. Na 3.2, por sua vez, são apresentados os resultados advindos da comparação por áudios, isto é, cada áudio de um subcorpus, C-ORAL-ESQ e C-ORAL-BRASIL, foi comparado com cada áudio de outro subcorpus. Quando relevantes ( $\mathrm{p}<0,05)$, o resultado do teste U de Mann Whitney é apresentado.

\subsection{Comparação de frequência e duração de pausas preenchidas por subcorpus}

A quantidade total de pausas preenchidas - 94 - bem como a duração total, pode ser visualizada na Tabela 2 . Nota-se que há 
mais pausas preenchidas tanto em relação à frequência de ocorrência quanto em relação à duração.

Na distribuição total das pausas preenchidas dos corpora, isto é, as pausas marcadas com TMT nas transcrições, o C-ORAL
-ESQ apresentou maior duração total do que o C-ORAL-BRASIL. Ambos os corpora apresentam distribuição assimétrica negativa razoavelmente semelhante (Figuras 1 - 2), com longa cauda à direita, com mais outliers no C-ORAL-BRASIL.

Tabela 2 - Duração e frequência de ocorrência de pausas preenchidas nos corpora

\begin{tabular}{c|c|c|c|c|c|c|c}
\hline Corpus & $\begin{array}{c}\text { Total } \\
\text { duração }\end{array}$ & mean & median & std & $\max$ & $\min$ & Qt. pausas \\
\hline C-ORAL-BRASIL & 23.964 & 0.533 & 0.432 & 0.336 & 1.801 & 0.070 & 45 \\
\hline C-ORAL-ESQ & 27.889 & 0.569 & 0.504 & 0.316 & 2.064 & 0.181 & 49 \\
\hline
\end{tabular}

Fonte: Elaborado pelo autor.

https://colab.research.google.com/drive/1UzHOvIaXhxkz3cwwCZLDa_vn1je5tWzE\#scrollTo=BTuVA8UC9_K\&line=1\&uniqifier $=1$

A maioria das pausas do C-ORAL-ESQ se gundos. O C-ORAL-BRASIL possui uma disconcentra em torno da média, entre 181 e tribuição um pouco menos homogênea, com 934 ms, com mais ocorrências de outliers em 1,311 segundos e a maior pausa preenchida encontrada no extremo direito, de 2,064 sea maioria das ocorrências entre $70 \mathrm{~ms}-\mathrm{a}$ menor pausa preenchida encontrada - e 935 ms, com outliers a partir de 1,109 segundos.

Figura 1 - Distribuição da duração das pausas preenchidas - TMT- no C-ORAL-ESQ.

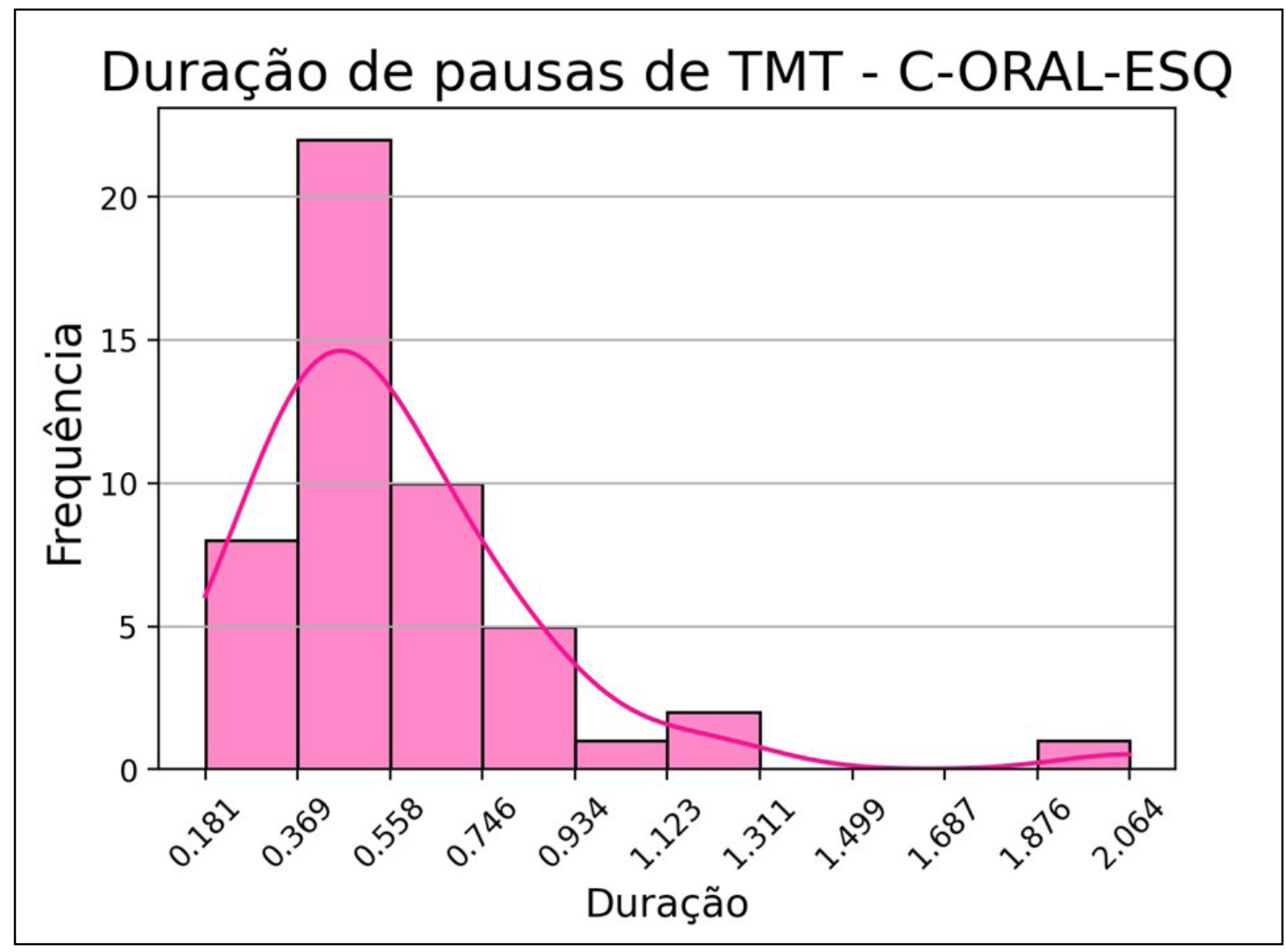


Figura 2 - Distribuição da duração das pausas preenchidas - TMT- no C-ORAL-BRASIL

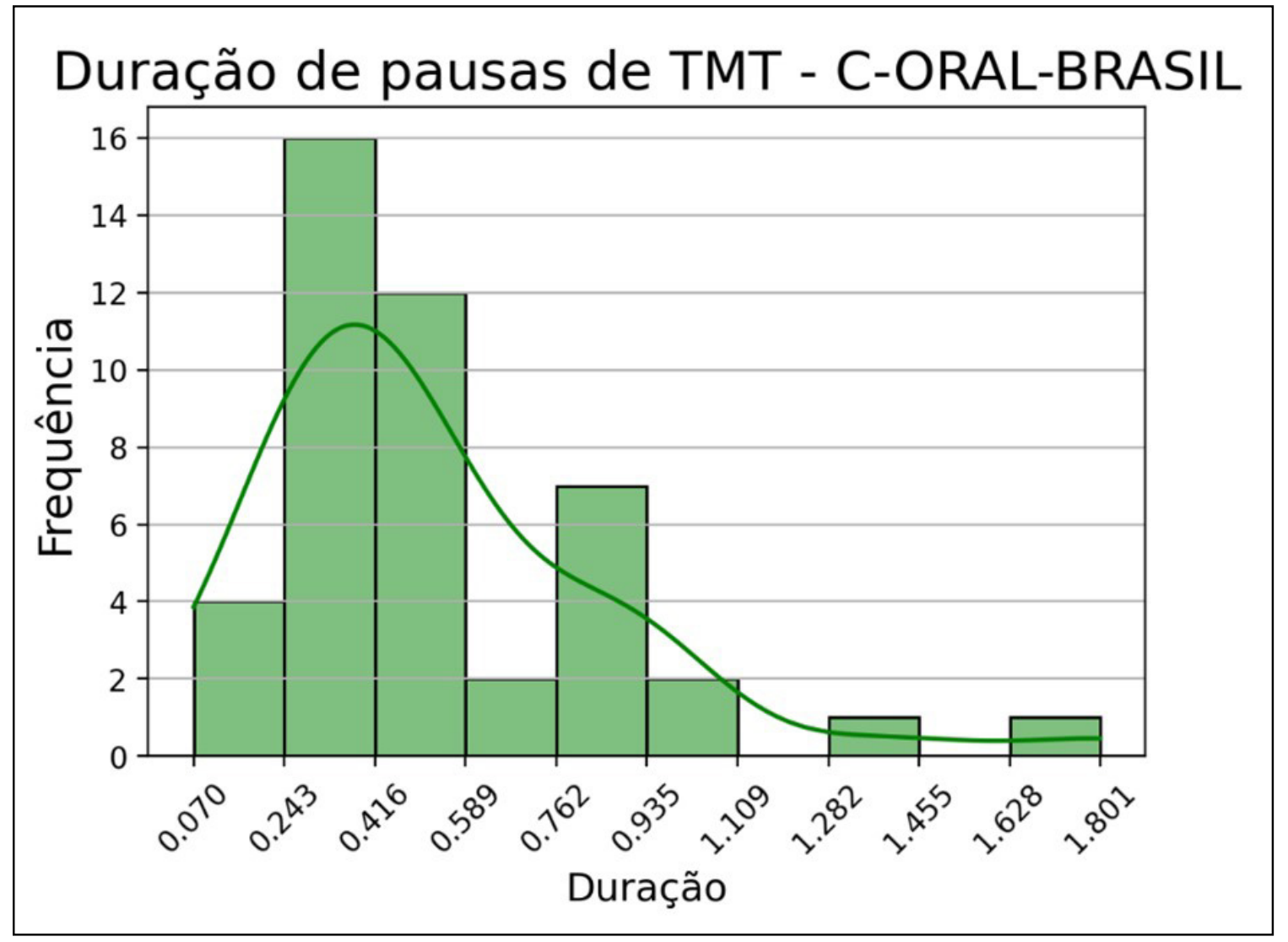

A qualidade vocálica dessas pausas preenchidas não consta na etiquetagem do corpus e seria necessária uma análise individual de cada ocorrência para classificá-las adequadamente.

\subsection{Pausas preenchidas por áudio}

Após a averiguação esquemática das pausas preenchidas de TMT em seções anteriores, é necessário verificar a distribuição dessas pausas por áudio. Na Tabela $3^{6}$, nota-se que a maior quantidade de pausas está concentrada no áudio med07, do C-ORAL-ESQ mas a duração das pausas de bfammn06 é maior, apesar da menor quantidade nesse último.

No boxplot da Figura 3, é possível verificar a duração dessas pausas. Nota-se que a maior média, representada pelo quadradinho branco nas caixas, é a do paciente do

6 Os audios do C-ORAL-BRASIL têm o prefixo bfammn e os do C-ORAL-ESQ o prefixo med. áudio med27, que além de possuir o maior outlier, próximo a 2 segundos, concentra a distribuição de suas pausas preenchidas em faixas de longa duração - a maioria no terceiro quartil - quando comparado com outros áudios do C-ORAL-BRASIL.

Na Figura 3, também é notável que o paciente de med27, apesar de ter realizado a maior quantidade de pausas preenchidas, realize hesitações menores do que a maioria dos pacientes - isso pode ser visualizado principalmente pela mediana ${ }^{7}$, que despreza os outliers presentes nesse áudio. Também chama a atenção que haja apenas 1 pausa preenchida no áudio med09, que aparece apenas como um risco nesse boxplot, e apenas 2 no áudio bfammn04. Outro ponto a ser destacado na Figura 2 é a mediana do participante do áudio bfammn05, a mais elevada

7 A mediana de um boxplot é o tracinho que corta transversalmente a caixa (quando a distribuição permite visualizar os quartis). 
do boxplot, o que indica que esse indivíduo possui maior constância na duração de suas pausas preenchidas, isto é, suas hesitações preenchidas são comumente de maior duração no áudio analisado.

A comparação entre a duração as pausas de TMT foi a única que produziu resultados estatisticamente significativos ${ }^{8}$ entre os participantes dos dois grupos.
Os resultados são relevantes para a comparação entre bfammn01 e med15 (p-value $=0,006) ;$ bfammn01 e $\operatorname{med} 20$ $(p$-value $=0,02) ;$ bfammn02 e med15 (p-value $=0,03) ;$ bfammn04 e med07 $(p$-value $=0,03) ;$ bfammn04 e med19 $(p$-value $=0,045) ;$ bfammn04 e med20 (p-value = 0,03); bfammn04 e med27 (pvalue $=0,06$ ).

Figura 3 - Distribuição da duração das pausas preenchidas por áudio

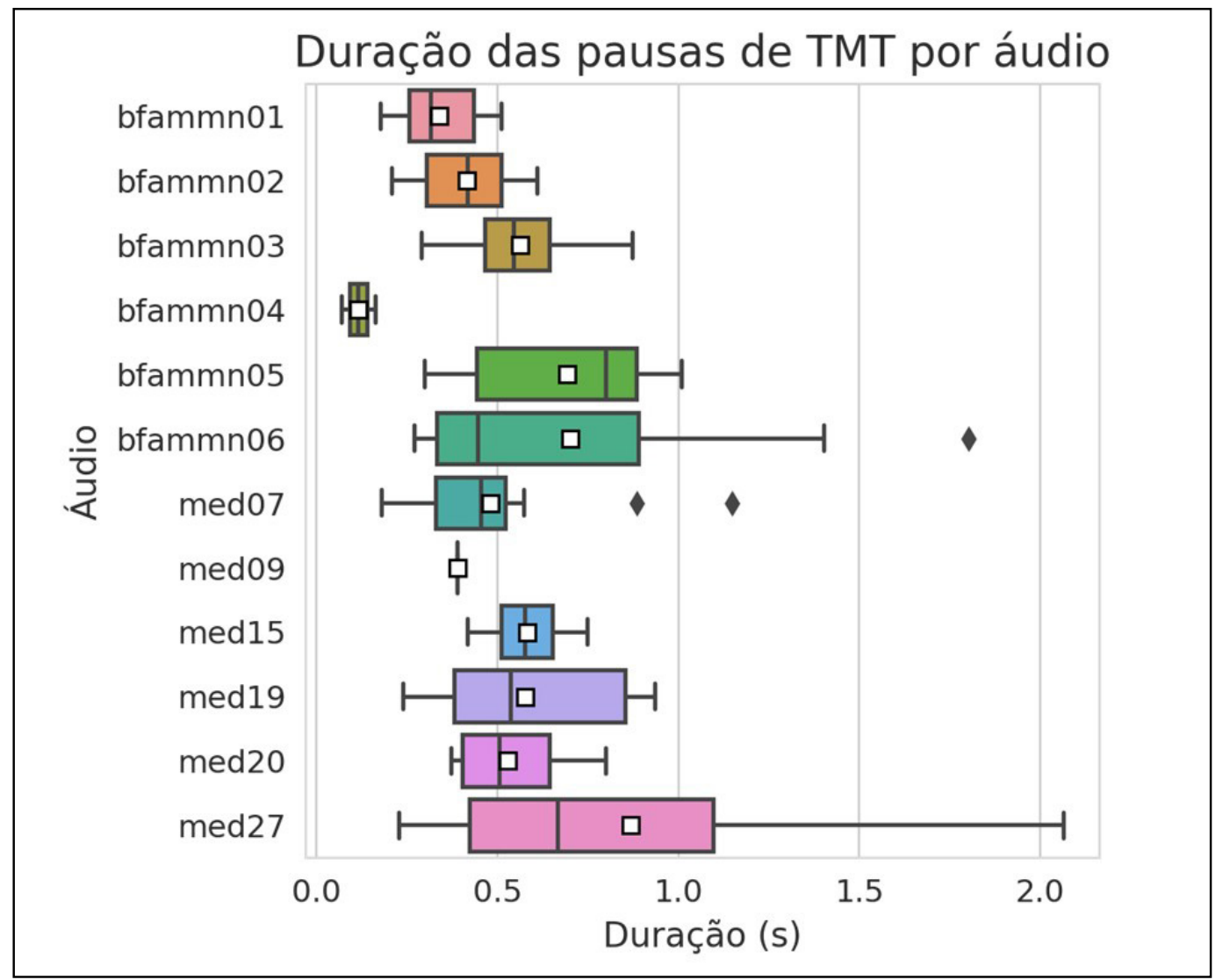

Tabela 3 - Duração e quantidade de pausas por áudio nos corpora -

\begin{tabular}{|c|c|c|c|c|c|c|c|}
\hline Áudio & Duração_total & mean & median & std & $\max$ & $\min$ & Qt_pausas \\
\hline med07 & 7.216 & 0.481 & 0.455 & 0.251 & 1.148 & 0.181 & 15 \\
\hline bfammn06 & 7.693 & 0.699 & 0.446 & 0.506 & 1.801 & 0.270 & 11 \\
\hline $\operatorname{med} 20$ & 5.811 & 0.528 & 0.504 & 0.153 & 0.800 & 0.373 & 11 \\
\hline bfammn02 & 4.151 & 0.415 & 0.417 & 0.141 & 0.611 & 0.207 & 10 \\
\hline bfammn05 & 6.932 & 0.693 & 0.799 & 0.259 & 1.008 & 0.299 & 10 \\
\hline
\end{tabular}

8 https://colab.research.google.com/drive/1lGn1VJ7f26BvVd5R5EWpdIpsX-LeaDOF?usp=sharing 
Tabela 3 - Continuação

\begin{tabular}{|c|c|c|c|c|c|c|c|}
\hline Áudio & Duração_total & mean & median & std & $\max$ & $\min$ & Qt_pausas \\
\hline med19 & 5.190 & 0.577 & 0.536 & 0.266 & 0.937 & 0.239 & 9 \\
\hline bfammn01 & 2.703 & 0.338 & 0.316 & 0.117 & 0.510 & 0.176 & 8 \\
\hline med15 & 4.072 & 0.582 & 0.577 & 0.113 & 0.748 & 0.418 & 7 \\
\hline $\operatorname{med} 27$ & 5.210 & 0.868 & 0.666 & 0.682 & 2.064 & 0.229 & 6 \\
\hline bfammn03 & 2.253 & 0.563 & 0.545 & 0.239 & 0.873 & 0.291 & 4 \\
\hline bfammn04 & 0.232 & 0.116 & 0.116 & 0.065 & 0.162 & 0.070 & 2 \\
\hline med09 & 0.390 & 0.390 & 0.390 & $\mathrm{NaN}$ & 0.390 & 0.390 & 1 \\
\hline
\end{tabular}

Fonte: Elaborado pelo autor

https://colab.research.google.com/drive/1UzHOvIaXhxkz3cwwCZLDa_vn1je5tWzE\#scrollTo=Sn709uWwmkW G\&line=1\&uniqifier $=1$

\section{Considerações finais}

Pacientes com esquizofrenia parecem hesitar mais na fala espontânea do que pessoas sem esquizofrenia. É possível que esses pacientes não tenham conseguido planejar adequadamente sua fala, dada a maior quantidade de pausas e maior duração das hesitações, e é necessário analisar individualmente a ocorrência de cada uma dessas pausas - em futuros trabalhos de viés mais qualitativo - para verificar se se tratam ou não de disfluências. Não é incomum que pausas preenchidas sejam utilizadas para manter o turno de fala, uma vez uma pausa silenciosa poderia sinalizar para outro falante - neste caso, o médico - de que o turno do paciente já poderia ser tomado.

Além disso, as pausas desses pacientes registraram maior duração total quando comparados em conjunto com os participantes do C-ORAL-BRASIL analisados. Quando comparados em conjunto, isto é, subcorpus com subcorpus, essas diferenças não foram estatisticamente relevantes.

Em relação à comparação individual realizada, isto é, cada participante de um sub- corpus comparado com cada participante do outro, foram registrados resultados variáveis, em geral, com maior quantidade de pausas e maior duração para pacientes com esquizofrenia do que os participantes sem esquizofrenia. Nessa etapa da comparação, foram registrados resultados estatisticamente relevantes apenas para duração das pausas no teste U de Mann Whitney.

\section{Referências}

ALPERT, Murray, KOTSAFTIS, Antonis, POUGET, Enrique. At Issue: speech fluency and schizoprenic negative signs. Schizophrenia Bulletin, vol. 23, número 2, 1997, pp. 171-177.

ALPERT, Murray, CLARK, Aileen, POUGET, Enrique. The syntatic role of pauses in the speech of schizophrenic patients with alogia. Journal of Abnormal Psychology, vol. 103, n. 4, 1994, pp. 750-757.

ANDREASEN, N. C. Positive and negative symptoms in schizophrenia. A critical reappraisal. Archives of General Psychiatry 1990, vol. 47, pp. 615-621.

ANDREASEN, N. C., \& Grove, W. M. Thought, language and communication in schizophrenia: Diagnosis and prognosis. Schizophrenia Bulletin, 12, 1986, pp. 356-359. 
ANDREASEN, N. C. A scale for the assessment of thought, language and communication (TLC). Schizophrenia Bulletin, 12, 1986, pp. 473-482.

BIBER, D. Representativiness in Corpus Design. Literary and Linguistic Computing, Vol. 8, No. 4, 1993. Disponível em: http://otipl.philol.msu. ru/media/biber930.pdf

BLACK, D; GRANT, JON. The essential companion to the diagnostic and statistical manual of mental disorders. Fifth edition. Washington D.C; Londres; American Psychiatric Publishing, 2014.

BOER, J. $\mathrm{N}$ de et al. Language in schizophrenia: relation with diagnosis, symptomatology and white matter tracts. Revista Nature, v. 10, 2020, pp. 1-10.

BORTFELD, $\mathrm{H}$ etal. Disfluency Rates in Conversation: Effects of Age, Relationship, Topic, Role, and Gender. Language and Speech, n.44(2), 2001, pp. 123-147.

BUTTERWORTH, B.L. Evidence for pauses in speech. In: Butterworth, B.L. (ed) Language Production, 1, Speech and Talk. Londres, London Academic Press, 1980.

ÇOKAL, Derya, et al. Disturbing the rhythm of thought. Speech pausing patterns in schizophrenia, with and without formal thought disorder. PLOS ONE, n. 14 (5), 2019, pp. 1-14.

COMPTON, M.; LUNDEN, A.; CLEARY, S. et al. The aprosody of schizophrenia: Computationally derived acoustic phonetic underpinnings of monotone speech. Schizophrenia Research, n. 197, 2018, pp. 392-399.

CONDRAY, R.; STEINHAUER, S. R.; VAN KAMMEN, D.P.; KASPAREK, A. The language system in schizophrenia: Effects on capacity and linguistic structure. Schizophrenia Bulletin, 28, 2002, pp. 475-490.

COVINGTON, M. A. et al. (2005). Schizophrenia and the structure of language: The linguist's view. Schizophrenia Research, 77, 85-98.

ESPOSITO, Anna et al. The significance of empty speech pauses: cognitive and algorithmic issues. The Significance of Empty Speech Pauses: Cognitive and Algorithmic Issues. In: Mele F., Ramella G., Santillo S., Ventriglia F. (eds) Advances in Brain, Vision, and Artificial Intelligence. BVAI 2007. Lecture Notes in Computer Science, vol 4729. Springer, Berlin, Heidelberg, 2007, pp. 542-554.
FELDSTEIN, S, JAFFE, J. Schizophrenic speech fluency : a partial replication and a hypothesis. Psychological Reports, n.13, 1963, pp-775-780.

LIDDLE, P. F., NGAN, E. T. C., CAISSIE, S.L. et al. Thought and language index: An instrument for assessing thought and language in schizophrenia. British Journal of Psychiatry, n. 181, 2002, pp. 326-330.

LIN, Y, DING, H, ZHANG, Y. Emotional prosody processing in schizophrenic patients: a selective review and meta-analysis. Journal of Clinical Medicine, n.7, 363, pp.1-26.

MATSUMOTO, K. et al. Frequency and neural correlates of pauses in patients with formal thought disorder. Frontiers in Psychiatry, 2013, vol. 4, article 127, pp. 1-9.

MORICE, R. D., \& Ingram, J. C. L. Language complexity and age of onset of schizophrenia. Psychiatry Research, 9, 1983, pp. 233-242,

KUPERBERG, G. Language in Schizophrenia. Part 1: An Introduction. Language and Linguistics Compass n.4, vol.8, 2010, pp. 576-589.

o'CONNEL, Daniel C., KOWAL, Sabine. Pausology. Computers in Language Research 2. Walter de Gruyter e CO, Berlim; Nova Iorque, 1983.

OGATA, J., GOTO, M., ITOU, K. The use of acoustically detected filled and silent pauses in spontaneous speech recognition. ICASSP 2009: IEEE International Conference on Acoustics, Speech and Signal Processing. Anais, pp. 4305-4308.

RASO, T.; MELLO, H. (Eds.). C-ORAL-BRASIL I: Corpus de referência do português brasileiro falado informal. 1. ed. Belo Horizonte: Editora UFMG, 2012.

ROCHA, B.N. O corpus C-ORAL-ESQ e a estrutura informacional da fala de pacientes com esquizofrenia. Working papers em Linguística, 20 (1), pp. 212-238.

STEUBER, L.C. Disordered Thought, Disordered Language: A corpus-based description of the speech of individuals undergoing treatment for schizophrenia (2011). Dissertations and Theses. Paper 63.

Recebido em: 25/05/2021

Aprovado em: 20/08/2021 